gentlemen; but many of you have seen the same phenomena in others. During the early part of the spring of this year, I was attending a child of seven years of age, with precisely the same symptoms. The disease ran a similar course, and the treatment was, in all respects, the same, with the exception that less opium was given. The illness in this case was much more protracted, and the exhaustion was greater. It was necessary to keep up the stimulant for a much longer period. The child had a narrow escape, but is now in good health; although a loud mitral bruit affords evidence of the serious damage which the heart has sustained.

On the Propriety of giving Stimulants in Acute Rheumatism. You must judge as to the propriety of giving or withholding stimulants from the general state of the patient, according to the same principles which enable you to decide as to the quantity and nature of the nourishment required. If you decide upon giving stimulants, you will determine the quantity after considering the strength of the pulse and heart's action and the condition of the nervous system. In some cases, you need not give any, and in others will give only very small quantities ; but there are cases which seem almost hopeless, that may be saved by the timely administration of small doses very frequently repeated; so that within two or three days, although a patient may have taken as many bottles of brandy, not the slightest indication of alcoholism has been produecd.

Practically, it is of great importance to regulate the quantity of alcohol according to the requirements of the system, and to administer it in small doses at regular intervals, so as to support the failing powers from hour to hour. No greater mistake could be made than to attempt to act upon any arbitrary rules in the use of this most valuable remedy. For instance, to regard alcohol as a necessary remedy to be employed, and to be depended upon, in every case of rheumatic fever or any other disease, would be as grave an error as to refuse to give it in any case. There are cases of acute rheumatism which will get well without a drop of alcohol. There are cases of the same malady in which it would be very wrong to give alcohol-at least, during the earlier period of the attack; and there are cases which I believe can be saved by this remedy alone. It is possible that alcohol, by its action on the blood and nervous system, may, in certain cases, tend indirectly to increase the tendency to what we term the inflammatory conditions; but it is quite certain that, in other cases, it diminishes the tendency to these changes; and it is beyond dispute that inflammation of some of the most important organs of the body will resolve, and the effused products be absorbed, while the patient is taking from ten to twenty ounces of brandy in the twenty-four hours. It is also true that the absorption of inflammatory products is carried on quite as rapidly in a low condition of the system under the influence of alcohol, as in a strong one in which not a drop of alcohol is given. Alcohol does not prevent the healing of wounds; but there are states of the system where repair does not take place until alcohol has been given to the patient.

The question as to whether alcohol should be given or withheld in a case is generally determined, not by the fact of the presence or absence of any special morbid process, but by the patient's powers at the

112 time; and in a case where alcohol is given, the proportion is determined by the degree of exhaustion. If the strength has diminished very rapidly, you would, from the first, give more than if the powers had been slowly failing. If the pulse diminished in frequency and increased in power after a few doses, you may be sure you are giving the right quantity. If the alcohol seems to produce little or no change you must give more. But if you watch a few cases from day to day, you will be more convinced of the great importance of studying the effects of this remedy in exhausting disease than from anything I can say on the matter.

\section{OAriginal Communications.}

\section{ON INFLAMMATION : ESPECIALLY ON PNEUMONIA.}

By Edward Copeman, M.D., Norwich.

[Read before the Norfolk and Norwich Pathological Socicty.]

THE age we live in is a wonderful age; a period in which such rapid strides have been made in all the arts and sciences as almost to confound man's understanding. Even during the lives of many here, great changes bave taken place in almost everything around us. The art of war is entirely different from what it was in the days of our youth; and in chemistry, the names familiar to us in our pupilage are supplanted by others, which are as Hebrew to those who have not continued the study of that science. This tendency to rapid progress has shown itself in our own profession also, and we are every now and then startled almost out of our propriety by the introduction of theories and opinions which well nigh upset the fixed rules and principles of our professional life. I shall not trouble you with any lengthened disquisition as to these circumstances; but it may be well to remark that it does not follow that every alteration is an improvement, or that every new theory is true. It may be right after all to employ antiphlogistic remedies in acute diseases, instead of trusting to brandy. It may be better to amputate a limb affected with synovial disease than merely to excise the diseased joint. It may prove true that the first sound of the heart is synchronous with the contraction of the ventricle, and the second coincident with and caused by the closing of the aortic valves; in which case we shall not have to unlearn all we have hitherto been taught upon the subject, and unsettle all our landmarks for the diagnosis of diseases of the heart. We must, after all, view with a certain degree of distrust all novel theories which materially entrench upon previously well recognised facts; although I would by no means refuse them a fair and un. prejudiced, examination, for generally, no doubt, something at least that is useful may be sifted out of them.

Inflammation is one of the principal subjects concern. ing which new views and novel modes of treatment are adopted. Nevertheless, more than half a century ago, John Hunter expressed many of the opinions we now hear, and $J$ question whether we at the present time understand the subject better than he did. He said "we have much less occasion for evacuations in inflammations than there was formerly, the lancet therefore in inflammation, and also purgatives, are much more laid aside." Again, "we may be said to live above par, at the full stretch of living, therefore, when disease attacks us, our powers cannot be excited further, and we sink, so as to require being supported and kept up to that mode of life to which we have been accustomed." And again, " in inflammation when the constitution is strong then it will be commonly the most manageable, for strength 
lessens irritability. It is probable that the blood of people of weak habits is weak in its living principle, which it therefore very soon loses upon extravasation, so as to become unfit for a bond of union, and degenerates into an extraneous body."

In discussions upon inflammation I think we ought to keep prominently before us the fact that the blood is a vital fluid, containing in itself the elements of all the tissues of the body; and, that it may be charged at warious times with more or less of one or other of these relements, according to the nature and quantity of nourishment taken, without its being, in the true sense of the word, diseased. When its chemical composition is altered, or it contains elements not natural to it in its healthy state, it may be said to be diseased; and in all inflammatory complaints both these conditions of the blood require to be recognised and distinguished. In inflammations, the blood may be either healthy or dis. eased; and the type and treatment of the inflammation will vary accordingly. In sthenic inflammations I believe it to be much more important to consider the vital properties, its power of coagulation, than the chemical composition of the blood. In asthenic, on the contrary, it would be a great boon to the profession if it were discovered to what extent and in what manner the latter characters of the blood are disturbed or modified.

Let us examine for a moment how this subject may be illustrated by pneumonia, a disease which has of late excited great interest in the profession, owing to the conflicting views and opinions entertained by different practitioners. In the sthenic form, pneumonia attacks for the most part those who seem to be in the enjoyment of good health, but who are plethoric, full of blood, and in fact verging upon any disease of which hyperæmia would be a predisposing cause. A person in this condition is exposed to some exciting cause of disturbance to his lungs, probably either cold or over-exertion. If the chest be long exposed to the direct influence of cold, the blood will be driven from the surface and accumulate in the internal organs; if over-exertion be the cause, the lungs will be overfilled with blood by increased action of the heart. This active congestion of the lungs produces more or less of shock to the system, and im. pedes the ready flow of blood through the vessels of the organ affected. This impediment is increased by the tenacity of the fibrine which such blood contains, and a free circulation cannot be again established until the blood is relieved by a deposit of some of its fibrine and an increase of its watery constituents. Thus fibrine is thrown out by nature to relieve the obstructed circulation, producing consolidation; and if the vis medicatrix be powerful enough to bear this temporary interruption to the vital processes, the deposit will be reabsorbed when the congestion no longer exists. Of course a good deal of agitation of the system will have accompanied these changes; and probably a natural diaphoresis or expectoration, or flow of urine, or diarrhœa (though in these cases seldom the latter), will materially assist the power with which our bodies are so mercifully endowed, that of constantly tending to restore the healthy balance when disturbed by disorder or disease. Now in such a case as this, nature will often accomplish a cure with the aid simply of diluent drinks, avoidance of heavy food, and keeping the surface at a uniform and perspiring temperature. But on the other hand, the cure would be much accelerated, and rendered more safe and certain by bloodletting at the outset; or by antimonial diaphoretics, and nitric ether to excite the action of the kid. neys, or both these combined. The former at once diminishes the column of blood, and, if adopted quite early, anticipates the effusion into the air-cells by rendering it unnecessary; the latter accomplishes the same purpose indirectly by means of the skin and kidneys, though not always in time to prevent a certain amount of deposit of fibrine in the lung. However, even in this case, provided the congestion is relieved, there will be no increase of effusion; and what has already taken place will either be naturally absorbed, or its absorption may be hastened and secured by the aid of mercurial action. Here again we arrive at a point in dispute at the present day, viz., the action of mercury in the treatment of inflammation. But setting aside theory for a moment, does any practitioner of experience doubt the power of mercury to rernove the products of inflammation? Can any one question the fact of its power to remove deposits of lymph and pus, who has witnessed its effects in iritis and hypopium? Here its effects are visible, definite, unmistakable. Can it be doubted that it produces the same effects in other organs? No-in these matters, experience must guide us; and I will fearlessly undertake to say that in cases of sthenic pneumonia, where the fibrinous deposit in the substance of the lungs requires to be quickly removed, the object will be obtained most effectually (if it can be obtained at all), by a discreet employment of mercury; and this, with less detriment to the constitution than would arise from the prolonged continuance of the pathological condition for which it is employed.

But how are we to deal with other kinds of pneumonic inflammations-asthenic-secondary-recurrent -lobular? For these occur usually in the course of fevers, or other diseases of debility, and do not depend upon hyperæmia, or upon a too highly fibrinated condition of the blood; they are, however, like the sthenic, dependent upon retarded circulation through the lungs, or in other words, congestion.

In many instances, as in fevers, the blood contains poisonous ingredients which diminish nerve force, and deprive any organ of the power of resisting exciting causes of disease.

With respect to the lungs, for instance, if cold be taken, there may not be nerve force enough to resist its effects, and congestion will be the consequence; but is this a kind of inflammation to require loss of blood? The quantity of blood is not in excess, but it lacks the healthy condition by which it stimulates the heart and arteries to propel it equally through the body; and the best way of relieving these asthenic local congestions is to excite additional nerve force by internal stimulants, such as ammonia, brandy, etc.

But there are also intermediate cases in which pneumonia is neither dependent entirely upon hyperæmia, nor upon poisoned blood; but in which the constitutional power is so slight and the natural resistance to the causes of disease so imperfect, that, on exposure to exciting causes, congestions and inflammations may take place simply for want of nervous power to resist them. In these cases, the blood, although not diseased, as in fevers, is neither superabundant, nor does it contain an inordinate quantity of fibrine, so as to require either blood.letting or the cutting off of supplies; and in such cases a combination of a diaphoretic treatment to relieve the pressure upon the lungs, and stimulants to force the blood through them without delay, will, I think, be found the most efficacious. I also believe that these last cases are less likely to recover of themselves, than the first I mentioned-the sthenic-for want of that natural vital power without which no severe dis. eased action can be overcome. In all three of these conditions-the sthenic, the asthenic, and that consequent upon diseased blood from fevers- the blood, when retarded in its circulation through the lungs, produces the same physical signs and symptoms: transudation into the air-cells, cough, dyspnœa, pain in the chest, pyrexia, coloured expectoration, and, at last, consolidation of the lung. But, although the symptoms are very similar, in each case enabling us to diagnose pneamonia, yet, as the causes of them and the early pathological conditions vary in each, so we may reasonably expect the necessity of varying the treatment accord- 
ingly. It would be taking a very narrow view of the question to say that, because the disease is pneumonia, we must therefore treat it by blood-letting, or we must not treat it by blood-letting, or it is better to let it alone, for it will get well of itself. We can have no such definite rule of practice as this in our profession; we must be guided in our treatment by well grounded general principles; and the great art we have to learn is to be able to modify those principles in detail to suit the varying exigences of those pathological conditions included in the general name of the disease.

If we are called early to a case of pneumonia, when the attack is sudden and severe, the patient young, plethoric, and not the subject of any constitutional disease, we should best relieve the patient, and save him the nlterior ehanges belonging to the disease, by early and decided blood-letting.

In asthenic cases, uncomplicated by other disease, I have often seen great relief afforded in the early stage by local blood-letting, aided by diaphoretics; taking care at the same time to give either tonics or stimulants, or both, should the general condition of the patient seem to require it.

In asthenic cases occurring in persons labouring under strumous and cachetic states of system, even local bleeding may be dangerous, and will be seldom if ever required; for, instead of being in excess and highly coagulable, the blood is poor and watery; and although it readily exudes, the consequent filling up of the air cells is less dangerous, and expectoration more easily takes place; we may safely take more time about the case, the patient will not be so early threatened with apnœa, and our surest remedies will be those which give tone to the general system, aided by counterirritation and medicines of diaphoretic and diuretic properties. In these cases, as well as in those which occur in fevers, there is less need of mercury to effect the absorption of the effused matter; for, containing less plastic material, it is more freely expectorated during the attack, and more easily absorbed afterwards, provided we have been able not only to relieve congestion but to give increased tone and nervous power to the whole body.

Where pneumonia has arisen secondarily in the course of typhus and other fevers of a low type, I have always found it the most successful practice to trust, as far as medicines are concerned, to the appropriate treatment of the fever generally; and to limit the special treatment of the local disease to fomentations and counterirritation by turpentine, blisters, or other stimulants.

The subject is far too large a one to be satisfactorily dealt with in a short paper on such an occasion as the present, where so little time can be spared for discussion; but I feel sure the hints I have given may be safely trusted in practice as a foundation for further observation and research.

\section{CASES OF PERFORATING ULCER OF STOMACH AND OF IN'TESTINE : WITH REMARKS.}

By Wм. Hinds, M.D., Professor of Botany at Queen's College, Birmingham; Lecturer on Botanical Science at the Birmingham Midland Institute.

[Read at the Midland Medical Association.]

[Concluded from p. 93.]

MY next case in some respects presents a most marked contrast to the one related. Instead of being a vigorous, healthy, and robust male, the patient was a feeble and delicate girl. In this case, too, the perforation was found not in the intestine, but in the anterior face of the stomach.

CASE II. Sarah A. B., aged 18, had suffered for some considerable time from pains in the stomach, and was in the habit, with a view to obtain relief from the pains, of taking a teaspoonful of black pepper. She was somewhat chlorotic in appearance; and the catamenia had been absent about eleven months. After tea on Friday, October $27 \mathrm{th}, 1818$, she was attacked with vomiting, pain of the stomach, and great depression. Subsequently, the same evening, she had several other attacks of vomiting. The vomiting recurred on the following morning; and, in addition, there were severe abdominal pains. Seen about midday, she was dressed and sitting at the table downstairs. Her tongue was slightly coated with brownish fur, and there was some feverishness complained of, and pains in the bowels, which were evidently tender; but, as she was dressed, a very accurate examination was not obtained. The pulse was feeble and small. The bowels bad not been moved since the previous day. She had taken in the morning some brandy and two teaspoonfuls of pepper. A threegrain calomel pill, combined with a grain of opium, was at once ordered, with a simple diaphoretic mixture; a sinapism was applied to the abdomen. Having gone to bed soon after the visit, she became gradually worse, and died at six the same evering.

Post Morten Examination thirty-seven hours after death. She was short in stature, well formed; but the mammary glands were not much developed. The ab: domen was much distended and tympanitic at the upper part. When it was cut into, much serous and watery liquid escaped; and about two quarts in all were removed from the peritoneal cavity. Some vascularity was observed upon the small intestines, and very small portions of lymph were seen in some parts. In the anterior face of the stomach, about an inch and a half below the cardia, a circular orifice was discovered, communicating with the interior. The size of the hole was about that of a large pea. Around the orifice, on the peritoneal surface, distinct appearances of inflammation existed; but there was no adhesion to adjacent parts. The whole parietes of the stomach were hypertrophied; and, indeed, appeared to be at least twice their normal thickness. The mucous surface was almost uniformly pale; and the excavation at the seat of the peritoneal laceration did not exhibit any vascularity, or differ in appearanee from the adjacent parts. The uterus was of the ordinary virgin calibre. The ovaries, externally congested in patches, contained several largely deve. loped Graafian vesicles, and some appearance of a falso corpus luteum. The other organs were bealthy.

REMARKs. No doubt, the question as to the cause or causes, and that as to remedies, if such there be, in these grave lesions, are the two points of inquiry which possess the highest interest, both to us as practitioners, and also to our patients; but there are besides several accessory considerations which well deserve some attention. On some of these points, I propose to make a few remarks; premising that I may do little more in some of these than merely specify the difficulties which present themselves.

We may ask, in the first place, what is the origin of a chronic perforating ulcer? One element in this inquiry would be, as to whether such a lesion would be favoured by delicacy of conformation or a low state of vital powet. In many such cases, as in Case II, there is this condition present.

But any free general conclusion in this direction must be seriously vitiated by the evident result of the chronic disease npon the stomach and its functions. A stomach with an irritable ulcer eannot be considered able to maintain its healthy operations; but if not, the failure strikes at the root of nutrition, and, as a consequence, of health. Now, even this conclusion may be considered in one sense to be falsified; for a most able author and authority declares that "almost all the patients have been young women, plump, and in good condition, who, up to the moment of the fatal seizure, either seemed to 